\title{
ZHX2 inhibits thyroid cancer metastasis through transcriptional inhibition of S100A14
}

\author{
Yankun Zhang ${ }^{1}$, Min Sun ${ }^{2}$, Lifen Gao ${ }^{1}$, Xiaohong Liang ${ }^{1}$, Chunhong Ma', Jinghui Lu ${ }^{2 *}$ and Xuetian Yue ${ }^{1,3^{*}}$ (D)
}

\begin{abstract}
Background: Thyroid cancer is the most common malignant endocrine tumour, and metastasis has become the main reason for treatment failure. However, the underlying molecular mechanism of thyroid cancer metastasis remains poorly understood. We investigated the role of the tumour suppressor zinc fingers and homeoboxes 2 (ZHX2) in the metastasis of thyroid cancer.

Methods: To study the role of ZHX2 in thyroid cancer metastasis, we evaluated the EMT process using cell migration, wound healing and lung metastatic tumour formation in vitro and in vivo models.

Results: ZHX2 expression was significantly decreased in thyroid cancer tissues, which correlated with poor prognosis of thyroid cancer patients. ZHX2 knockdown significantly promoted the migration of thyroid cancer cells. Mechanistically, ZHX2 associated with the S100 calcium binding protein A14 (S100A14) promoter to decrease the transcription of S100A14. Moreover, S100A14 was highly expressed in human thyroid cancer samples, and its expression negatively correlated with ZHX2 expression.

Conclusions: Inhibition of S100A14 attenuated the ZHX2 knockdown-induced enhanced metastasis of thyroid cancer cells both in vitro and in vivo. The evidence presented here suggests that ZHX2 inhibits the progression of thyroid cancer by blocking S100A14-mediated metastasis.
\end{abstract}

Keywords: ZHX2, Thyroid cancer, Metastasis, S100A14

\section{Background}

Thyroid cancer is the most common malignant endocrine tumour [1]. The incidence of thyroid cancer has been steadily rising in recent years (an approximately $4 \%$ increase annually), and it represents the most rapidly increasing malignancy among all major cancers, tripling in the past 3 decades [2]. Most thyroid cancer cases

\footnotetext{
*Correspondence: jinghuilu@email.sdu.edu.cn; yuexu@sdu.edu.cn ${ }^{1}$ Key Laboratory for Experimental Teratology of Ministry of Education, Key Laboratory of Infection and Immunity of Shandong Province and Department of Immunology, School of Basic Medical Sciences, Cheeloo Medical College of Medicine, Shandong University, Jinan 250012, China

${ }^{2}$ Department of Hernia and Abdominal Wall Surgery, General Surgery, Qilu Hospital, Cheeloo College of Medicine, Shandong University, Jinan 250012, China

Full list of author information is available at the end of the article
}

originate in the follicular epithelium and can be divided into papillary thyroid cancer (PTC), follicular thyroid cancer (FTC) and anaplastic thyroid cancer (ATC) according to the pathologic type [3]. Patients diagnosed with thyroid cancer at an early stage have an excellent prognosis. However, individuals with large, invasive tumours and/or distant metastasis have a 5-year survival rate of only $\sim 40 \%[4,5]$. Thus, there is a need to better understand the molecular causes of thyroid cancer metastasis to develop new treatment options.

Zinc fingers and homeoboxes 2 (ZHX2) is a transcription factor belonging to the ZHX family that was initially identified based on its natural mutation in $\mathrm{BALB} / \mathrm{cJ}$ mice $[6,7]$. As a regulator of the oncofoetal genes alpha foetal protein (AFP) and glypican-3 (GPC3), ZHX2 inhibits the development of hepatocellular carcinoma [8-10]. ZHX2 is widely expressed and participates in many types of original author(s) and the source, provide a link to the Creative Commons licence, and indicate if changes were made. The images or other third party material in this article are included in the article's Creative Commons licence, unless indicated otherwise in a credit line to the material. If material is not included in the article's Creative Commons licence and your intended use is not permitted by statutory regulation or exceeds the permitted use, you will need to obtain permission directly from the copyright holder. To view a copy of this licence, visit http://creativecommons.org/licenses/by/4.0/. The Creative Commons Public Domain Dedication waiver (http://creativeco mmons.org/publicdomain/zero/1.0/) applies to the data made available in this article, unless otherwise stated in a credit line to the data. 
cancers, such as gastric cancer, lung cancer and clear cell renal cell carcinoma [11-13]. Recently, whole transcript microarray expression profiling detected the common expression of ZHX2 in brain metastatic PTC and primary brain tumours [14], suggesting that ZHX2 may play a role in thyroid cancer metastasis. However, whether and how $\mathrm{ZHX} 2$ is involved in the progression of thyroid cancer is unknown.

Metastasis is the major obstacle for thyroid cancer treatment. Mounting evidence has demonstrated that extracellular matrix (ECM) degradation plays an important role in cancer metastasis [15-17]. S100 proteins, a family of ECM proteins related to metastasis, are a group of multigene calcium-binding proteins [18]. Among them, S100A14 (S100 calcium binding protein A14), a member of the S100 family of proteins, has received more attention in tumour progression. S100A14 has been reported to be dysregulated in various types of tumours and involved in the proliferation, apoptosis and signal transduction of tumour cells [19]. In particular, S100A14 regulates the epithelial-mesenchymal transition (EMT) process to influence the metastasis of prostate cancer and cervical cancer [20,21]. Further study clarified that S100A14 drives CCL2/CXCL5 signalling to promote breast cancer metastasis [22]. Until now, the role of S100A14 in thyroid cancer metastasis has not been elucidated.

To study the role of ZHX2 in thyroid cancer metastasis, we evaluated the EMT process using both in vitro and in vivo models. We found that overexpression of ZHX2 significantly restrained the thyroid cancer metastatic process, inhibiting wound healing and the migration of thyroid cancer cells. Mechanistically, ZHX2 inhibited S100A14 transcription. The expression of S100A14 negatively correlated with ZHX2 expression in human thyroid tumour specimens. More importantly, knockdown of S100A14 attenuated ZHX2 silencing-induced thyroid cancer cell migration both in vitro and in vivo. Therefore, targeting ZHX2 and S100A14 signalling may be a useful strategy to inhibit thyroid tumour progression.

\section{Materials and methods}

\section{Patients and the clinical samples}

Forty-nine thyroid tumour tissues and thirty-four paratumour tissue samples were collected from Qilu Hospital, Shandong University (Additional file 1: Table S1). All of the patients had undergone surgery without preoperative radiation or chemotherapy before 2020 .

\section{Cell lines and reagents}

The human ATC cell lines 8305C (RRID: CVCL_1053), BHT101 (RRID: CVCL_1085) and KMH-2 (RRID: CVCL_S641) were kindly provided by the Stem Cell
Bank, Chinese Academy of Sciences (Shanghai, China). The PTC cell line BHP10-3 (RRID: CVCL_6278) was obtained from Qilu Hospital of Shandong University [23]. All cell lines that were authenticated using STR profiling within the last 3 years were included. All experiments were performed with mycoplasma-free cells. They were maintained in Dulbecco's modified Eagle's medium (DMEM) (Thermo Fisher Scientific, C11995500CP) or minimum essential medium (MEM) (Thermo Fisher Scientific, 11095080) supplemented with $10 \%$ foetal bovine serum (FBS) (Biological Industries, 04-001-1A), $100 \mathrm{U} /$ $\mathrm{mL}$ penicillin, $100 \mathrm{mg} / \mathrm{mL}$ streptomycin (Solarbio, P1400) and $2 \mathrm{mM} \mathrm{L}$-glutamine (Solarbio, G0200) and incubated at $37{ }^{\circ} \mathrm{C}$ in an incubator with $5 \% \mathrm{CO}_{2}$ and saturated humidity. The ZHX2-expressing vector pcDNA3-ZHX2HA has been described previously [24]. Polymerase chain reaction (PCR)-amplified human S100A14 was cloned into the pcDNA3.0 vector. The primers used for cloning are shown in Additional file 2: Table S2. The siRNAs against ZHX2 and S100A14 (Additional file 2: Table S2) were synthesized by the GenePharma Inc. (Shanghai, China). These siRNAs were transfected into cells using the Lipofectamine ${ }^{\mathrm{TM}} 2000$ Transfection Reagent (Invitrogen, 11668019). After incubatation for $48 \mathrm{~h}$, the cells were collected for RNA or protein extraction.

\section{Quantitative real-time PCR (RT-qPCR)}

Total RNA of cells and tissues was extracted using TRIzol reagent (TIANGEN Biotech, DP424) and reverse transcribed into cDNA with a Revert Aid First Strand cDNA Synthesis Kit (Thermo Fisher Scientific, K1622). Quantitative PCR (qPCR) was carried out using a BioRad C1000 Thermal Cycler CFX96 Real-Time System with ChamQ Universal SYBR qPCR Master Mix (Vazyme Biotech, Q711). The primers used in this article are shown in Additional file 2: Table S2.

\section{Western blotting}

Thyroid tissues were homogenized in cell lysis buffer (Beyotime, P0013), and protein extracts were quantified by BCA protein assays (Beyotime, P0009). Equal amounts of proteins were loaded in SDS-polyacrylamide electrophoresis gels, transferred to Immobilon-P membranes (Millipore, Billerica) and incubated overnight at $4{ }^{\circ} \mathrm{C}$ with the following primary antibodies: mouse antiGAPDH (Proteintech, 60004, 1:5,000), rabbit anti-ZHX2 (Proteintech, 20136-1-AP, 1:2,000), rabbit anti-S100A14 (Proteintech, 10489-1-AP, 1:500), rabbit anti-N-cadherin (Proteintech, 22018-1-AP, 1:2000), rabbit anti-E-cadherin (Proteintech, 20874-1-AP, 1:5000) rabbit anti-vimentin (Proteintech, 10366-1-AP, 1:2000), and mouse antibeta actin (Proteintech, 66009-1-Ig, 1:5000). The membranes were washed with PBST 3 times and subsequently 
incubated with secondary HRP-conjugated anti-mouse (Proteintech, SA00001-1, 1:5000) or anti-rabbit IgG secondary antibodies (Proteintech, SA00001-2, 1:5000). The signal was detected by enhanced chemiluminescence (ECL) reagent (Millipore, WBULS0500) using the Tanon Bio-Imaging Systems (Tanon, 4600).

\section{Immunohistochemical staining assay}

Thyroid cancer tissues were fixed in $4 \%$ formaldehyde solution to make paraffin sections. The deparaffinized sections were permeabilized with $0.3 \%$ Triton X-100 and blocked with $5 \%$ bovine serum albumin (Solarbio, C1032) following incubation with antibodies against ZHX2 (Proteintech, 20136-1-AP) and S100A14 (Proteintech, 104891 -AP) overnight at $4{ }^{\circ} \mathrm{C}$. The slips were then incubated with secondary antibodies and detected by DAB staining. Images were captured by microscopy (Olympus, Japan). Each section was counted independently by three pathologists and scored according to the staining intensity (no staining $=0$; weak staining $=1$; moderate staining $=2$; strong staining $=3$ ) and the number of stained cells $\quad(0-5 \%=0 ; \quad 6-25 \%=1 ; \quad 26-50 \%=2 ; \quad 51-75 \%=3$; $76-100 \%=4)$ [25]. The final score was calculated using the following formula: intensity $\times$ stained number.

\section{Migration assay}

Briefly, $2 \times 10^{4}$ thyroid cancer cells with or without ZHX2 overexpression in $200 \mu \mathrm{L}$ of serum-free medium were added to the upper chambers of the Transwell plate, and the lower chambers were filled with $800 \mu \mathrm{L}$ of medium containing $10 \% \mathrm{FBS}$ as a chemoattractant. After $24 \mathrm{~h}$, the migrated cells were fixed in $4 \%$ paraformaldehyde and stained with $1 \%$ crystal violet, and the nonmigratory cells on the upper surface of the chambers were removed by a cotton swab. Then, at least four random fields were selected and observed by microscopy (Olympus, Japan).

\section{Wound-healing assays}

Wound healing assays were performed in 6-well plates with confluent cells, and scratches were generated using $200 \mu \mathrm{L}$ pipette tips. The wells were then washed three times with FBS-free medium and cultured for an additional $24 \mathrm{~h}$, followed by the assessment of the relative wound closure areas using microscopy (Olympus, Japan).

\section{Luciferase reporter assay}

The S100A14 gene promoter region was cloned into the pGL3-basic vector. The firefly luciferase reporter pGL3S100A14 vector and Renilla luciferase plasmid (pRL-TK) were transiently co-transfected into the cells transfected with the pcDNA3-ZHX2 or control vector. pRL-TK was used for normalizing the transfection efficiency. Luciferase activities were measured by using a dual luciferase reporter assay kit (E1960, Promega). Primers for plasmid construction are shown in Additional file 2: Table S2.

\section{chromatin-immunoprecipitation (ChIP) assay}

ZHX2 overexpression vector (pcDNA3-ZHX2-HA)-transfected $8305 \mathrm{C}$ cells were harvested for the ChIP assay by using the EZ-Magna ChIP $^{\mathrm{TM}}$ A/G Chromatin Immunoprecipitation Kit (Millipore, 17-10086). Briefly, cells were fixed and sonicated to shear DNA to 200-1000 bp. The proteinDNA complexes were immunoprecipitated with anti-HA antibody (Proteintech, 51064-2-AP) or control IgG. The DNA fragments were collected and purified according to the manufacturer's protocol. qPCR was performed using the primers shown in Additional file 2: Table S2.

\section{Animal experiments}

BALB/c nude mice (6-7 weeks of age) were provided by Charles River and maintained under specific pathogenfree (SPF) conditions. ZHX2 knockdown or control 8305C cells were transduced with viral supernatants containing a recombinant lentivirus vector $\mathrm{LV}$-shNC or LV-shS100A14, respectively. Lentiviruses expressing shRNAs against ZHX2 and shS100A14 were purchased from Shanghai GenePharma. The cells were collected, and the knockdown efficiency was verified by western blotting. Then, 8305C cells $\left(1 \times 10^{6}\right)$ with ZHX2 and S100A14 knockdown individually or simultaneously were injected into $B A L B / c$ nude mice via the tail vein. On the 60th day of the experiment, mice were sacrificed, the total lung was weighed and lung tissues were collected for haematoxylin and eosin (H\&E) assays. Randomly selected fields of H\&E-stained lung tissues were captured. Images were captured by microscopy (Olympus, Japan). The number of migrated clones and migrated areas were calculated using Image).

All animals were killed by the inhalation of carbon dioxide. Putting the animals into a closed transparent container, carbon dioxide was injected into the container at a rate of $20 \%$ per minute until the animals died.

\section{Statistical analysis}

Statistical analysis was carried out with GraphPad Prism 8 software. Data are presented as the mean \pm standard deviation (SD). One-way ANOVA or Student's $t$ test was used to assess statistical significance. Statistical significance was reported as highly significant using $*(p<0.05), * *(p<0.01)$ or $* * *(p<0.001)$.

\section{Results}

Low expression of ZHX2 correlates with poor prognosis in thyroid cancer

To determine the protein levels of ZHX2 in thyroid tumours, we employed western blotting for collected thyroid tumour samples. As shown in Fig. 1A, 
adjacent non-tumour tissues (referred to as $\mathrm{P}$ ) showed higher levels of ZHX2 protein than thyroid cancer tissues (referred to as $\mathrm{T}$ ). Consistently, significantly lower expression of $Z H X 2$ mRNA in tumours was confirmed by RT-qPCR analysis (Fig. 1B). To further evaluate the location and expression of $\mathrm{ZHX} 2$ protein on thyroid cancer cells, we detected ZHX2 protein in thyroid cancer tissues using immunohistochemistry (IHC). We observed ZHX2-positive staining (arrow) in the nuclei of adjacent normal cells and ZHX2-negative or weak ZHX2-positive staining in tumour cells, as exemplified in Fig. 1C. Further semi-quantitative scoring showed that ZHX2 protein levels were significantly higher in adjacent normal tissues than in tumour tissues (Fig. 1D). The results of the Kaplan-Meier curve (http://kmplot.com) revealed that low expression of ZHX2 correlated with poor prognosis of thyroid cancer patients (Fig. 1E). Together, these results show that ZHX2 expression is decreased in thyroid cancer.

\section{$\mathrm{ZHX} 2$ inhibits the migration of thyroid cancer in vitro}

To further investigate the role of ZHX2 in thyroid cancer metastasis, we measured the migration ability of thyroid tumour cell lines with modulated levels of ZHX2. First, ZHX2 protein levels were detected in BHP10-3, BHT101, 8305C and KMH-2 cell lines. As shown in Fig. 2A, ZHX2 protein levels were lower in BHT101 and $\mathrm{KMH}-2$ than in BHP10-3 and 8305C cell lines. Therefore, we overexpressed ZHX2 in BHT101 and KMH-2 cells and knocked down ZHX2 in BHP10-3 and 8305C cells to evaluate the migratory ability. Vimentin, $\mathrm{N}$-cadherin and E-cadherin are well-known markers that indicate cell migration [26]. Here, we observed that ZHX2 protein levels negatively correlated with the protein levels of vimentin and $\mathrm{N}$-cadherin, and positively correlated with the protein levels of E-cadherin (Fig. 2B), indicating that ZHX2 restrains the migration of thyroid cancer cells. To verify this hypothesis, Transwell assays were performed. As displayed in Fig. $2 \mathrm{C}$, the migrated cell numbers were lower in ZHX2-overexpressing $\mathrm{KMH}-2$ and BHT101

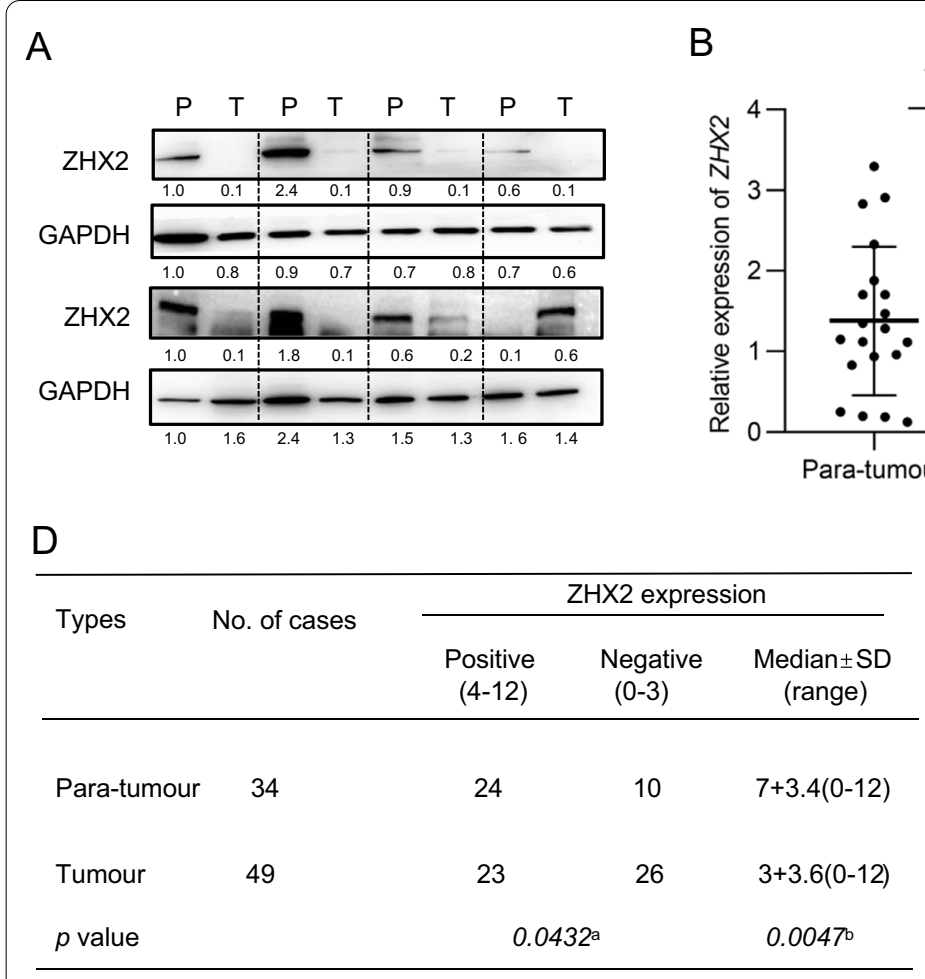

${ }^{\text {a }} \mathrm{p}$ value was studied by $\mathrm{CHI}$-sequare test, ${ }^{\mathrm{b}} \mathrm{p}$ value was studied by $t$ test
B

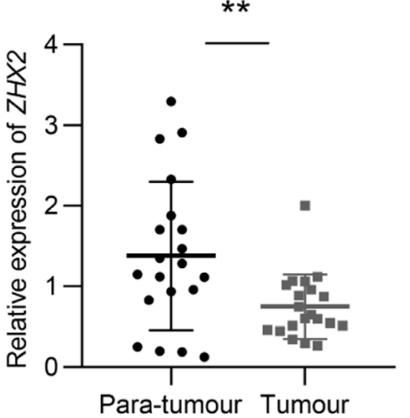

C

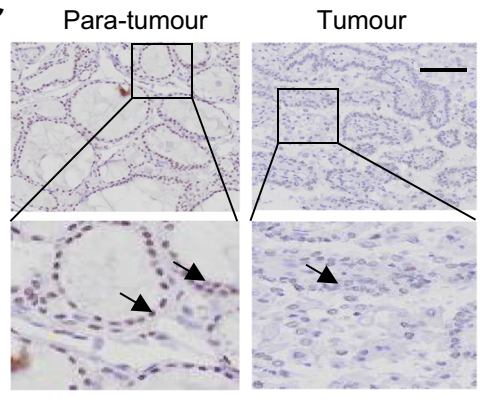

E

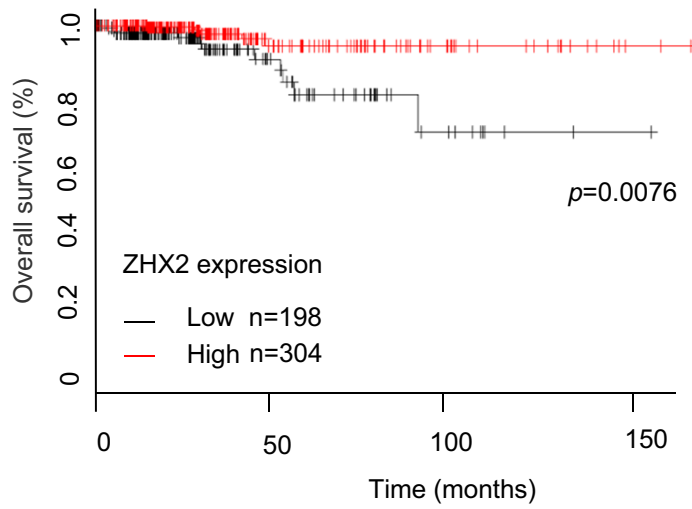

Fig. 1 Low expression of ZHX2 was associated with poor prognosis in thyroid cancer. A ZHX2 expression was measured in human tissue samples using western blotting (WB). GAPDH was used as the internal control. Thyroid tumour tissues (T); adjacent non-tumour tissues (P). B ZHX2 mRNA levels were evaluated by RT-qPCR in human specimens. ${ }^{* *} p<0.01$, the $p$ value was determined by Student's $t$ test. C Representative images of ZHX2 immunostaining in thyroid adjacent non-tumour and thyroid tumour tissues (Image magnifications of $100 \times$, bar: $100 \mu \mathrm{m}$ ). D Statistical analysis of ZHX2 expression in thyroid adjacent non-tumour and thyroid tumour tissues. Significant differences were determined by the chi-squared test. $\mathbf{E}$ Kaplan-Meier survival curves of thyroid cancer patients with ZHX2 expression were analysed by online software 

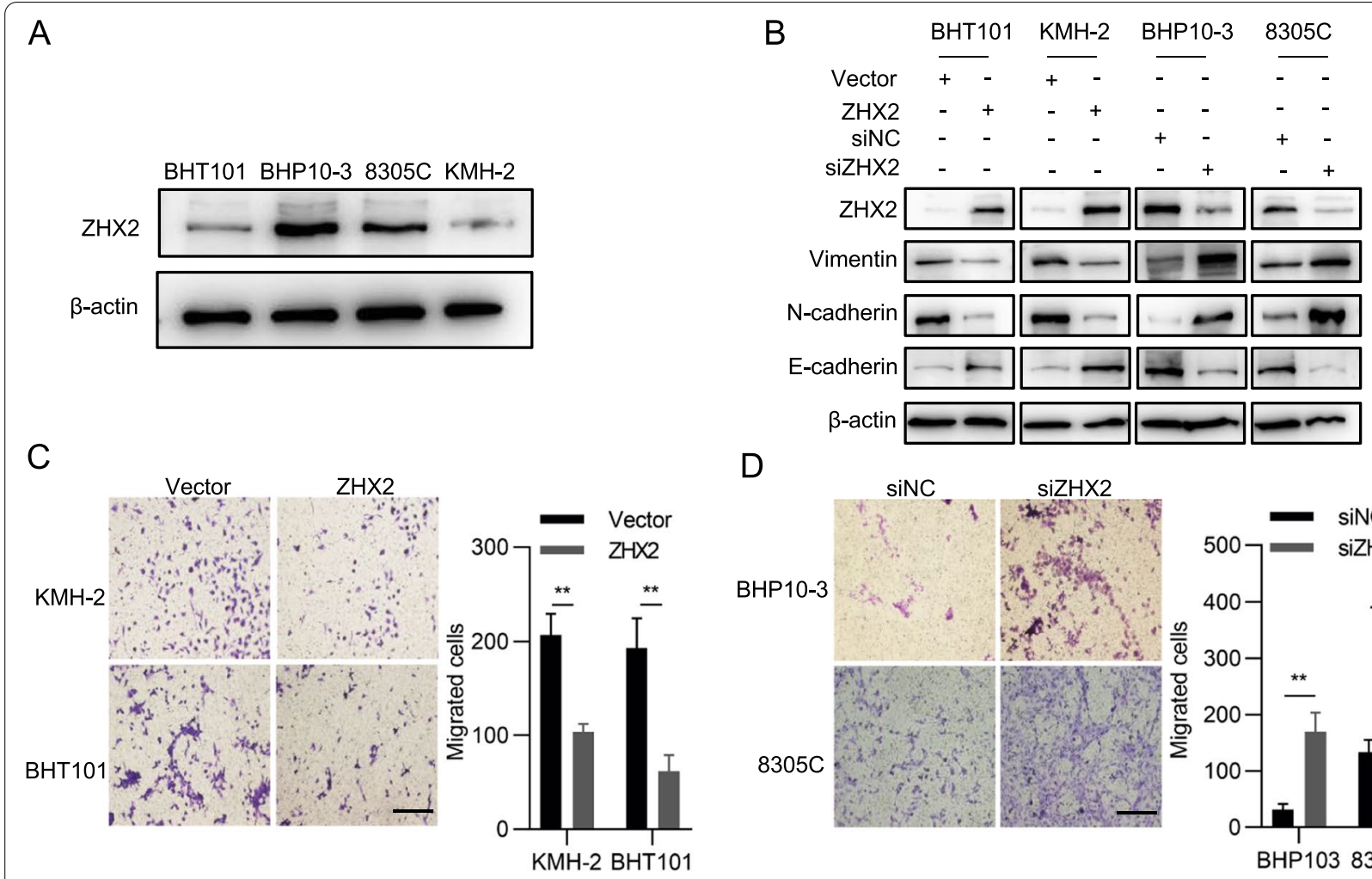

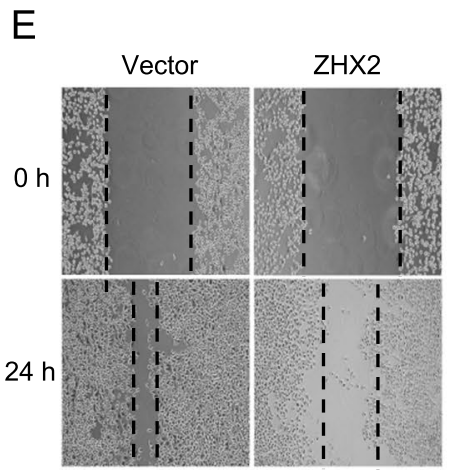

$\mathrm{KMH}-2$

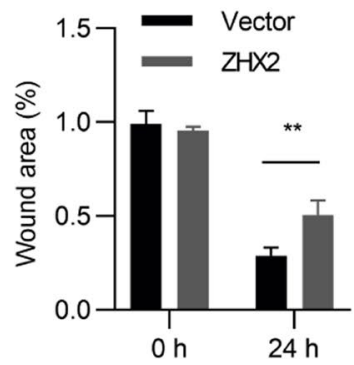

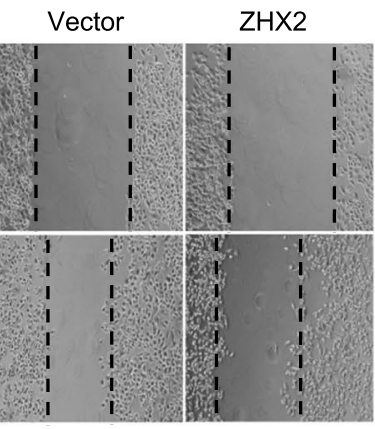

BHT101

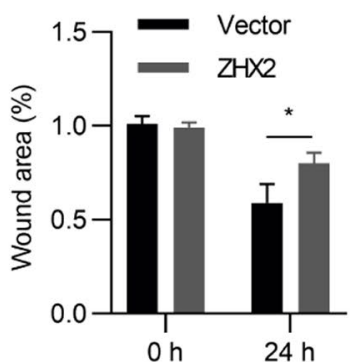

D



Fig. 2 ZHX2 restrains the migration of thyroid cancer cells. A The protein levels of ZHX2 in BHT101, BHP10-3, 8305C and KMH-2 cells were examined by WB. B EMT-related proteins N-cadherin, E-cadherin and vimentin were assayed in ZHX2-overexpressing KMH-2 and BHT101 cells or ZHX2-knockdown BHP10-3 and 8305C cells by WB. Thyroid cancer cells with the indicated manipulation were used to determine the migratory ability by using Transwell $(\mathbf{C}, \mathbf{D})$ and wound healing assays $(\mathbf{E})$. The migrated cells were photographed. Image magnifications: $100 \times(\mathbf{C}, \mathbf{D}), 40 \times(\mathbf{E})$. Bar: $200 \mu \mathrm{m}(\mathbf{C}, \mathbf{D}), 500 \mu \mathrm{m}(\mathbf{E})$. Representative pictures are shown. Data are presented as the mean \pm SD. Student's $t$ test, ${ }^{*} p<0.05,{ }^{* *} p<0.01$ 
cells than in control cells. Knockdown of ZHX2 by siRNAs increased the migration ability of both BHP10-3 and 8305C cells (Fig. 2D). Further wound healing assays also showed that cell motility was reduced in ZHX2-overexpressing thyroid cancer cell lines and increased in ZHX2 knockdown thyroid cancer cell lines (Fig. 2E). Above all, the data demonstrate that $\mathrm{ZHX} 2$ restrains the migration of thyroid cancer cells.

ZHX2 negatively correlates with S100A14 in thyroid cancer ECM degradation is an important biological process of metastasis [15-17]. The S100 protein family, belonging to ECM proteins, is closely related to tissue repair and tumour progression and has drawn much clinical attention in recent years [27-29]. Based on these findings, we evaluated the functional interaction between ZHX2 and S100 family members. Interestingly, through analysis of publicly available cBioPortal data, we detected a negative correlation of $\mathrm{ZHX} 2$ with multiple members of S100 (Additional file 3: Table S3). Among them, S100A14, on the top of the list, showed a significant negative correlation with ZHX2 expression (Fig. 3A). Consistent with publicly available data, a negative correlation between ZHX2 and S100A14 mRNA expression (Pearson's $r=-0.3912$ ) was also presented in our clinical samples (Fig. 3B). Furthermore, we also observed that the protein level of ZHX2 was negatively correlated with the S100A14 protein level in both thyroid cancer tissues and adjacent non-tumour tissues, as exemplified in Fig. 3C. These results indicate that ZHX2 inhibits the expression of S100A14 in thyroid cancer. Then, we aimed to determine the role of S100A14 in thyroid cancer. Notably, it was found that thyroid cancer tissues had elevated S100A14 mRNA levels compared with adjacent
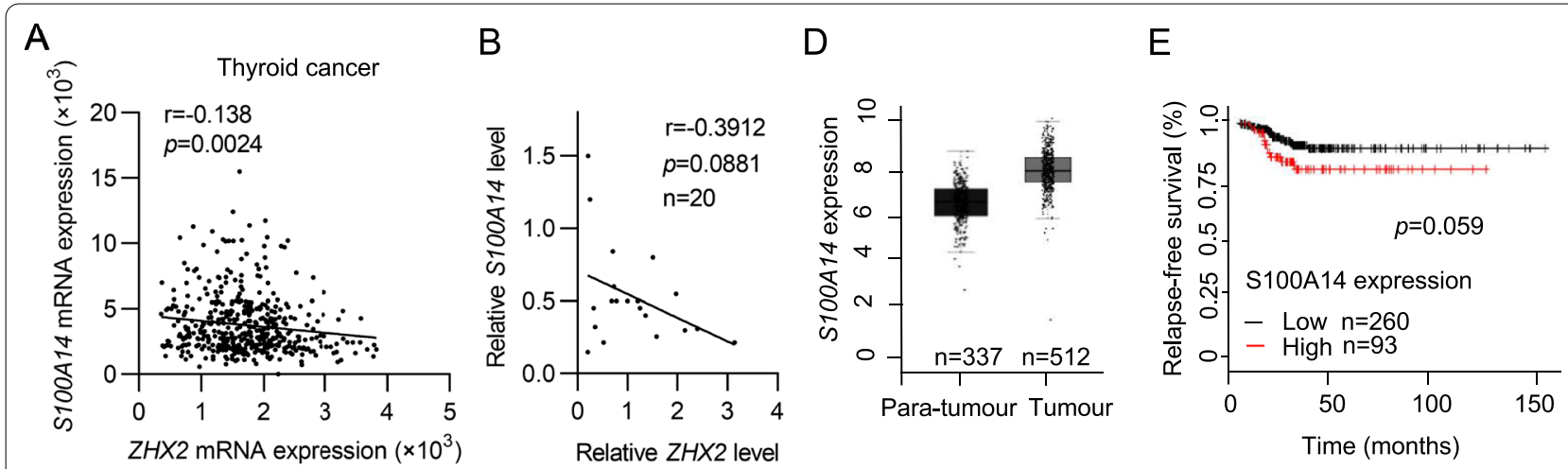

C

$\mathrm{ZHX} 2$
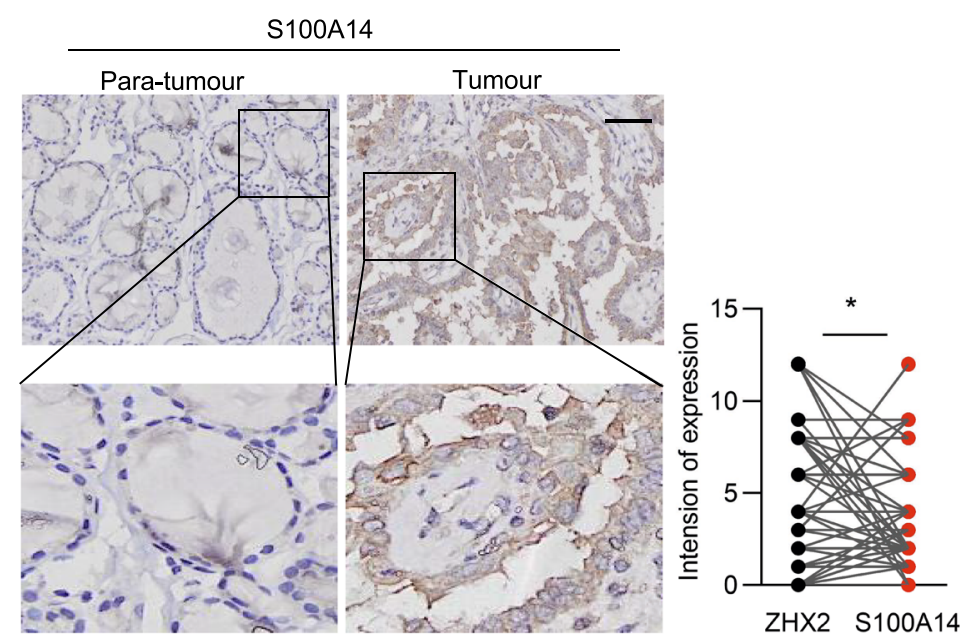

Fig. 3 ZHX2 inhibits S100A14 expression in thyroid cancer. A Correlations between ZHX2 and S100A14 mRNAs were analysed using the cBioPortal thyroid cancer dataset. B RT-qPCR analysis was used to determine the relationship between ZHX2 and S100A14 mRNAs in human specimens. C ZHX2 and S100A14 immunostaining was performed in thyroid para-tumour tissues and thyroid tumour tissues. Representative images are displayed (Image magnifications of $100 \times$, bar: $100 \mu \mathrm{m}$, left). Statistical analysis of ZHX2 and S100A14 correlation in samples (right). D S100A14 expression was determined in thyroid para-tumour tissues and thyroid tumour tissues from GEPIA datasets. E Kaplan-Meier relapse-free survival of thyroid cancer patients with S100A14 is presented. Data are presented as the mean \pm SD. Student's $t$ test, ${ }^{*} p<0.05$. A, B Pearson's correlation coefficients $(r)$ and $p$ values $(p)$ for two-sided correlation tests are shown 
non-tumour tissues by analysing GEPIA (http://gepia. cancer-pku.cn) (Fig. 3D). More importantly, KaplanMeier curves revealed that high expression of S100A14 correlated with poor prognosis in thyroid cancer patients (Fig. 3E), suggesting that S100A14 is an oncogene of thyroid cancer. Therefore, these data show that ZHX2 might inhibit thyroid cancer via S100A14.

\section{ZHX2 binds to the S100A14 promoter to repress its transcription}

Since ZHX2 is a well-known transcription factor, we examined S100A14 mRNA levels in thyroid cancer cell lines with ZHX2 manipulation. ZHX2 overexpression vector or siRNAs were transfected into thyroid cancer cell lines. RT-qPCR analysis showed that S100A14 mRNA levels were dramatically decreased in ZHX2overexpressing KMH-2 and BHT101 cells. Reciprocally, knockdown of ZHX2 obviously elevated the mRNA level of $5100 A 14$ in $8305 \mathrm{C}$ and BHP10-3 cells (Fig. 4A), indicating that ZHX2 might repress S100A14 expression at the transcriptional level. To verify this hypothesis, we performed dual luciferase and chromatin immunoprecipitation (ChIP) assays. We successfully cloned the S100A14 promoter region $(-1800$ to $+466 \mathrm{bp})$ in the pGL3-basic plasmid (pGL3-S100A14) and verified its promoter activity (Fig. 4B). The results of cotransfection and dual-luciferase assays showed that enforced expression of ZHX2 greatly inhibited S100A14 promoter activity in $8305 \mathrm{C}$ cells (Fig. 4C). Furthermore, a ChIP assay was performed in pcDNA3-ZHX2-HA-transfected $8305 \mathrm{C}$ cells to analyse the occupancy of ZHX2 in the promoter of S100A14. Bioinformative analysis identified four putative ZHX2 binding motifs on the S100A14 promoter by the Eukaryotic Promoter Database EPD (http://epd. vital-it.ch) [30, 31]. As shown in Fig. 4D, the locations of four putative $\mathrm{ZHX} 2$ binding sites on the promoter

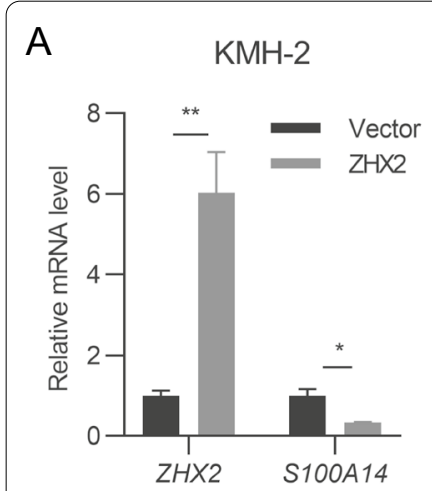

B
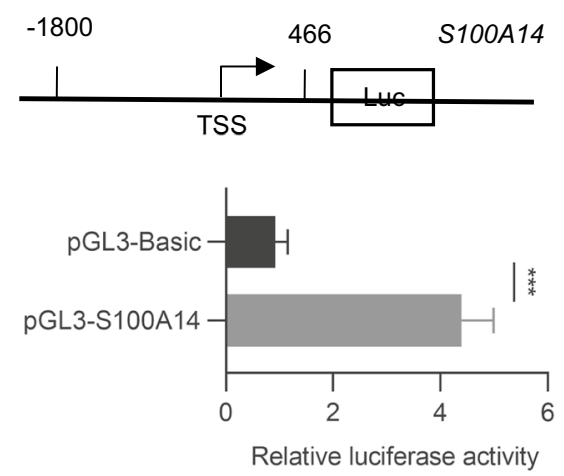

\section{BHT101}

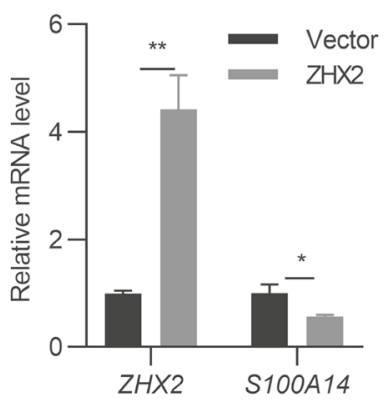

$8305 \mathrm{C}$

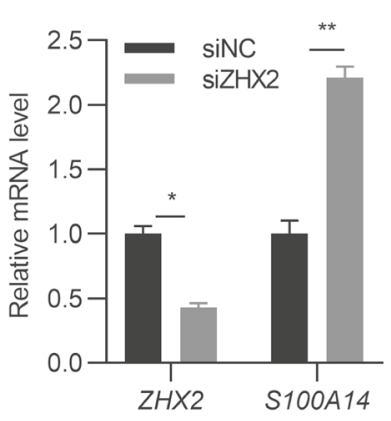

\section{BHP10-3}

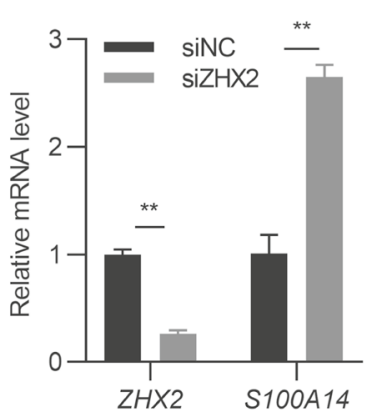

C
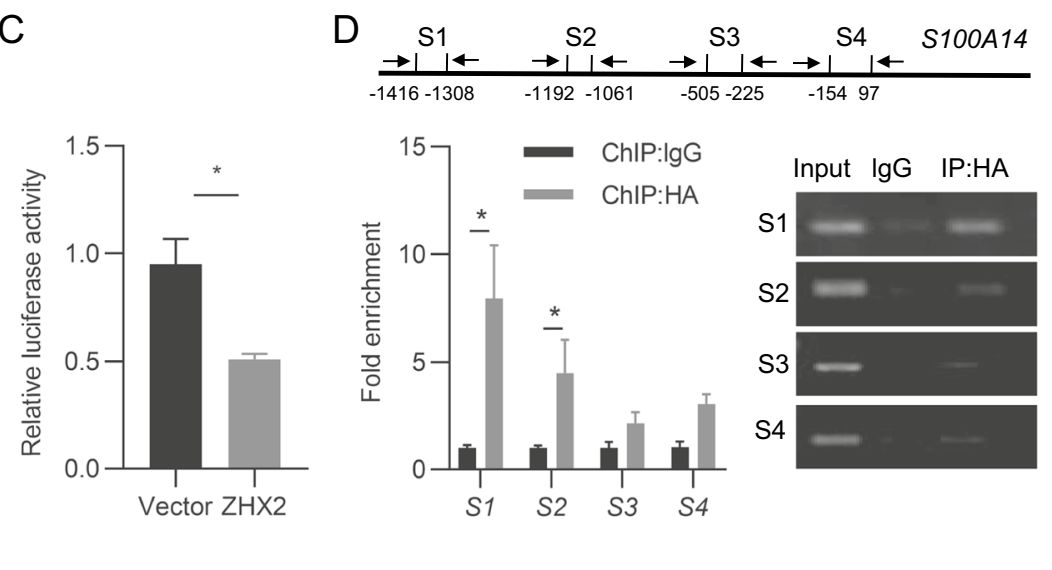

Fig. 4 ZHX2 binds to the S100A14 promoter to repress its expression. A RT-qPCR analysis of ZHX2 and S100A14 mRNA levels in thyroid cancer cells with ZHX2 overexpression or knockdown. B Diagram of the S100A14 promoter region and S100A14 promoter luciferase constructs are presented in the top panel. The promoter activity of PGL3-S100A14 was verified by the dual luciferase assay (lower panel). C S100A14 promoter activity was measured in $8305 \mathrm{C}$ cells with or without ZHX2 overexpression using a dual fluorescent reporter assay. D The ChIP assay was used to analyse ZHX2 occupancy on the promoter of S100A14. 8305C cells were transfected with a plasmid encoding ZHX2. HA antibody was used to pull down DNAprotein complexes in these cells. Primers targeting S100A14 promoter regions (S1-S4) are shown in the top panel. The lower panel presents the quantification of qPCR (left) and semiquantitative images (right). Data are presented as the mean \pm SD. Student's $t$ test. ${ }^{*} p<0.05,{ }^{* *} p<0.01,{ }^{* * *} p$ $<0.001$ 
region of S100A14 are presented. Among them, section 1 ( -1416 to $-1308 \mathrm{bp}$ ) is the major ZHX2 binding site, as section 1 primers induced significantly increased fold enrichment in the qPCR assay and an obviously amplified band in the semiquantitative PCR assay. Taken together, these data show that ZHX2 transcriptionally suppresses the expression of S100A14.

\section{ZHX2 restrains thyroid cancer cell migration via repression of $\mathrm{S} 100 \mathrm{~A} 14$ in vitro}

To verify whether the inhibition of thyroid cancer migration by ZHX2 was achieved by repressing S100A14, we performed the following experiments. As shown in Fig. 5A, overexpression of ZHX2 inhibited cell migration, and ectopic expression of S100A14 promoted cell migration. More importantly, ectopic expression of S100A14 significantly abolished the ZHX2-mediated inhibitory effect on cell migration in $\mathrm{KMH}-2$ cells. Consistently, ectopic expression of S100A14 eliminated the ZHX2-induced inhibition of wound healing in KMH-2 cells (Fig. 5B). To verify these results, we performed Transwell and wound healing assays using BHP10-3 cells with ZHX2 and/or S100A14 knockdown, simultaneously or independently. The efficiency of S100A14 knockdown in BHP10-3 cells was verified by RT-qPCR (Fig. 5C). Cell migration and the wound healing ability were increased in ZHX2 knockdown BHP10-3 cells. Notably, this inhibition was reversed when siS100A14 was cotransfected with siZHX2 (Fig. 5D, E). In accordance, western blot analysis revealed that ZHX2 protein levels were negatively associated with the protein levels of vimentin and $\mathrm{N}$-cadherin, positively correlated with E-cadherin. S100A14 protein levels were positively associated with vimentin and $\mathrm{N}$-cadherin proteins, negatively correlated with E-cadherin. Cotransfection of S100A14 with ZHX2 eliminated the ectopic expression of ZHX2induced decreases in vimentin and $\mathrm{N}$-cadherin proteins, and increases in E-cadherin proteins in $\mathrm{KMH}-2$ cells (Fig. 5F). Reciprocally, silencing S100A14 abolished the accumulation of vimentin and $\mathrm{N}$-cadherin proteins in ZHX2 siRNA-transfected BHP10-3 cells (Fig. 5G). Above all, ZHX2 inhibits thyroid cancer migration via S100A14.

\section{ZHX2 inhibits lung metastatic tumour formation of thyroid cancer cells via S100A14}

To further confirm that ZHX2 restrains thyroid cancer metastasis via S100A14, we performed lung metastasis assays in mouse models. Stable cell lines with varied ZHX2 and S100A14 levels were established through transduction of $8305 \mathrm{C}$ with ZHX2-shRNA and/ or S100A14-shRNA viruses, respectively. As shown in Fig. 6A, endogenous ZHX2 or S100A14 was efficiently knocked down by ZHX2-shRNA or S100A14-shRNA, respectively. Then, four groups of cells (shNC, shZHX2, shS100A14 and shZHX2/shS100A14) were injected into nude mice via the tail vein to evaluate the formation of lung metastatic tumours (Fig. 6B). The results showed that ZHX2 knockdown increased the lung weight and promoted the formation of lung metastatic tumours, displayed as heavier lungs, larger migrated areas and more metastatic tumours in the shZHX2 group than in the shNC group. Knockdown of S100A14 markedly decreased the lung weight, migrated area and number of metastatic tumours compared to those of the shNC-transduced group. More importantly, S100A14 knockdown abolished the ZHX2 silencinginduced enhancement of lung weight and lung metastasis (Fig. 6C-F). Therefore, these data demonstrate that ZHX2 inhibits thyroid cancer metastasis via S100A14 in in vivo mouse models.

\section{Discussion}

As an endocrine malignancy, thyroid cancer is commonly seen in the clinic [32]. Although thyroid cancers such as PTC have a favourable prognosis and a low death rate, a certain number of patients develop more aggressive forms that are unresponsive to radioactive iodine and chemotherapy, resulting in increased incurability and patient morbidity and mortality [33]. For these reasons, the identification of key molecules responsible for thyroid cancer metastasis is urgent and highly demanded for improving the clinical outcome. In this study, we found that low expression of ZHX2 led to upregulation of S100A14, which in turn promoted cell migration, wound healing and lung metastatic tumour formation. These results suggest that ZHX2 is a key regulator of thyroid cancer metastasis, which transcriptionally represses S100A14 expression to inhibit thyroid cancer metastasis. Therefore, strategies aiming to manipulate ZHX2 and S100A14 might be helpful to treat thyroid cancer.

Identified as a transcription factor, ZHX2 inhibits the development of hepatocellular carcinoma (HCC). Previous reports, including ours, showed that ZHX2 represses $\mathrm{HCC}$ oncogenes, such as AFP, GPC3, cyclin A/cyclin E and MDR1, which are involved in many biological processes of HCC development, including cell proliferation, cell migration and chemoresistance [7, 8, 10, 34]. However, the role of ZHX2 in thyroid cancer has not been reported. Here, we found that low expression of ZHX2 was associated with poor prognosis in patients with thyroid tumours. Further study showed that silencing ZHX2 increased cell migration and wound healing in thyroid cancer cell lines. In line with our findings, overexpression of ZHX2 reduced the migratory ability of lung cancer cells via the p38MAPK signalling pathway [11]. However, a report showed that $\mathrm{ZHX} 2$ promotes the migration 


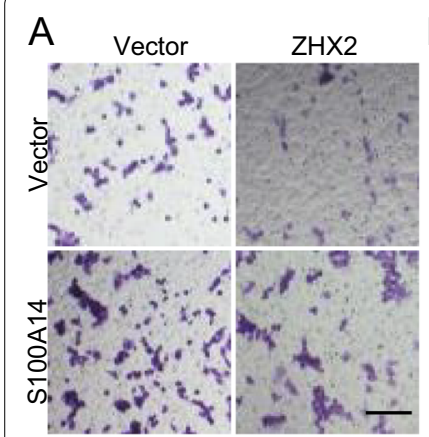

$\mathrm{KMH}-2$

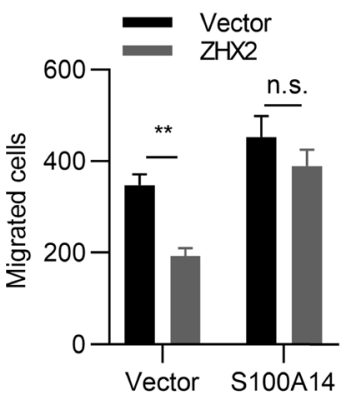

C

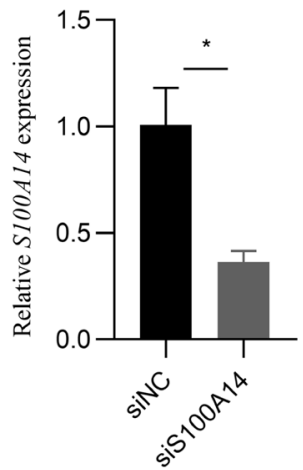

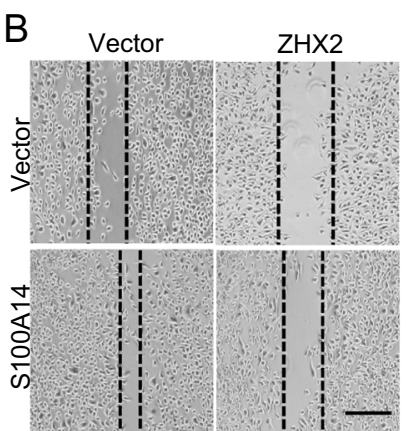

$\mathrm{KMH}-2$



Transfection $24 \mathrm{~h}$



BHP10-3

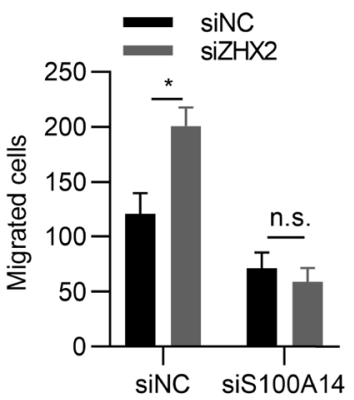

E

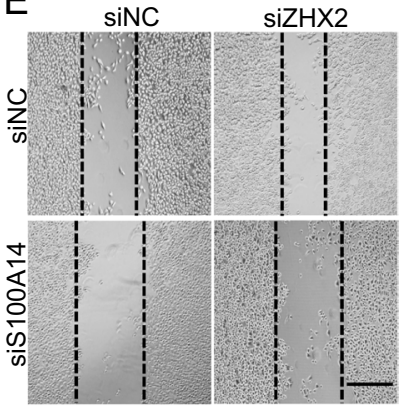

BHP10-3

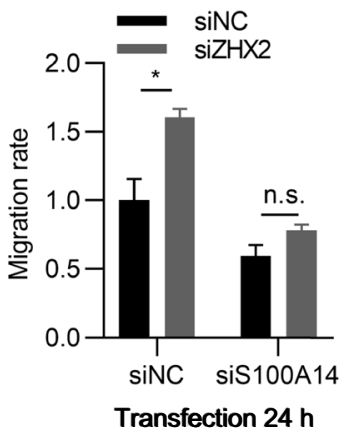

F

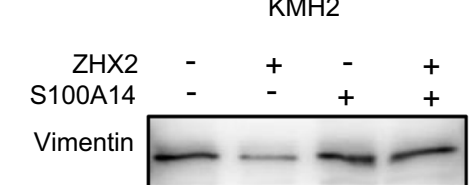

$\mathrm{N}$-cadherin

E-cadherin

$\beta$-actin
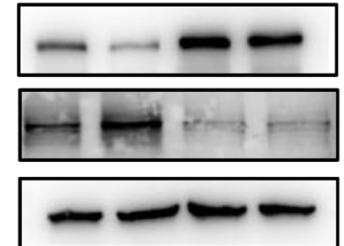

G

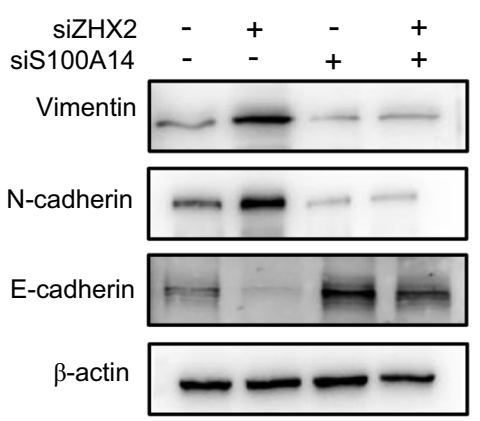

Fig. 5 ZHX2 restrains thyroid cancer cell migration via repression of S100A14 in vitro. A-F ZHX2-overexpressing or ZHX2-knockdown KMH-2 and BHP10-3 cells were transfected with S100A14 or siS100A14, respectively. Transwell assays (A, D), wound healing assays (B, E) and protein levels of EMT markers N-cadherin, E-cadherin and vimentin $(\mathbf{F}, \mathbf{G})$ were assessed in these cells. The migrated cells were photographed. Image magnifications: $200 \times(\mathbf{A}, \mathbf{D}), 40 \times(\mathbf{B}, \mathbf{E})$. Bar: $100 \mu \mathrm{m}(\mathbf{A}, \mathbf{D}), 500 \mu \mathrm{m}(\mathbf{B}, \mathbf{E})$. C RT-qPCR analysis for knockdown of S100A14 in BHP10-3 cells were shown. Data are the mean \pm SD. One-way ANOVA. ${ }^{*} p<0.05,{ }^{* *} p<0.01$, ns not significant

of clear cell renal carcinoma cells [35]. Therefore, ZHX2 might play different roles in tumour metastasis depending on the tumour type. Previous study reported that ZHX2 is widely expressed in many tissues, such as liver, lung, kidney and brain [36]. Thus, understanding the role of ZHX2 in different types of cancers will be beneficial for cancer-targeting therapy.

One of the important questions is how ZHX2 inhibits the metastasis of thyroid cancer. Based on bioinformatics analysis, we found that ZHX2 negatively correlated with S100A14 in thyroid cancer. As a member of the S100 family, S100A14 (also known as breast cancer membrane protein 84 ) is a newly identified member of the S100 protein family and has recently gained significant attention in cancer research [37]. Mounting evidence has shown that S100A14 is involved in many biological processes of cancer development, including cell proliferation, apoptosis, cell migration and cell differentiation [19, 38-40]. 


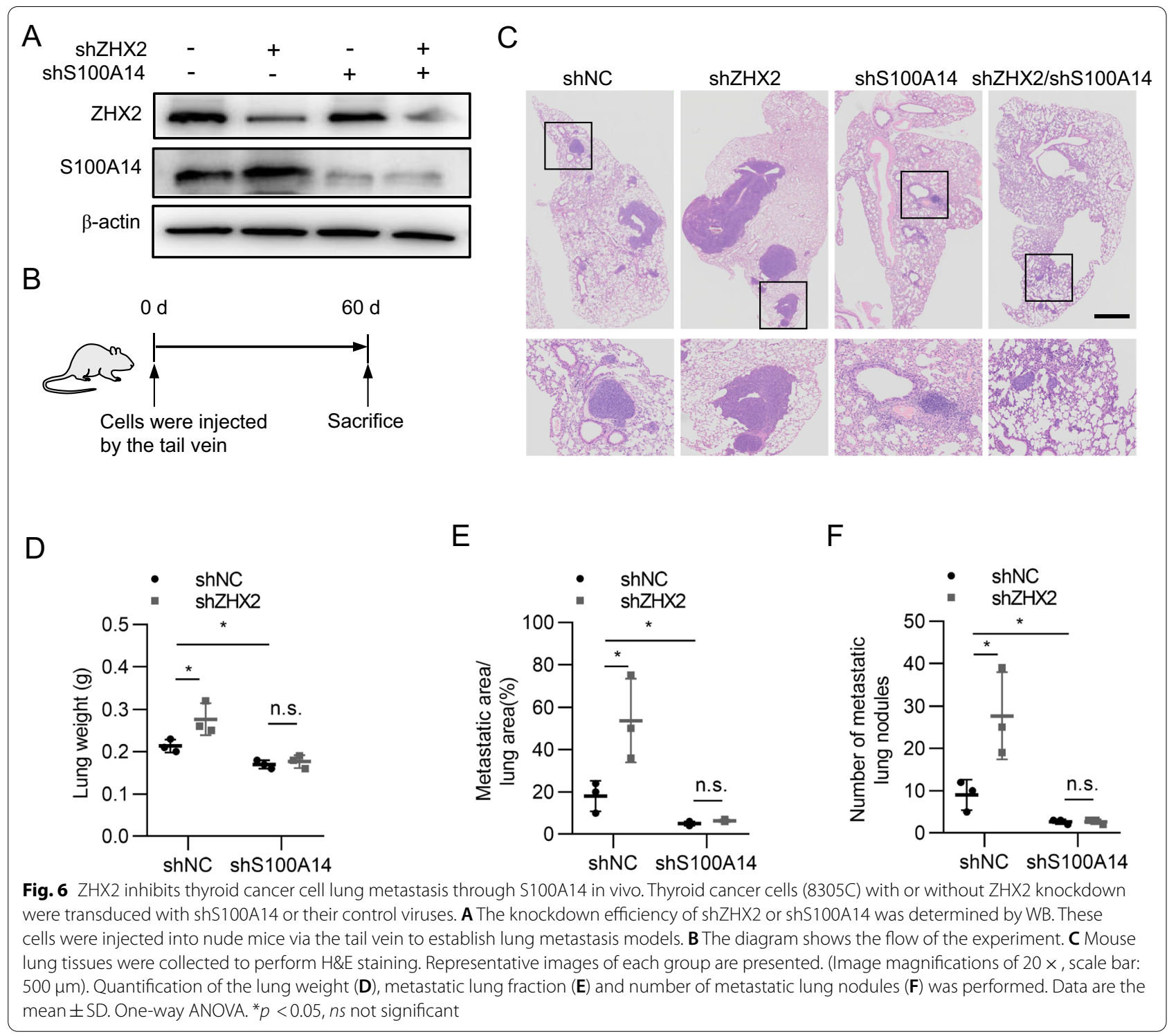

In particular, it is involved in the EMT processes of cell migration and invasion, which are associated with the outcome of thyroid cancer [41, 42]. S100A14 belongs to extracellular matrix proteins that are involved in the biological process of EMT [20, 43]. Here, we found that knockdown of S100A14 abolished ZHX2 silencinginduced cell migration both in vitro and in vivo, demonstrating that $\mathrm{ZHX} 2$ is involved in thyroid cancer metastasis through S100A14. More importantly, our data illustrated that ZHX2 bound to the S100A14 promoter to repress its transcription. Consistent with our findings, many reports have shown that ZHX2 inhibits the promoter activity of many oncogenes, such as AFP, GPC3, MDR1 and Cyclin A/E [8-10, 34]. Further studies showed that ZHX2 binds to specific DNA sequences through interaction with NF-YA or NF- $\mathrm{kB}$, depending on the tissue context $[13,44]$. Here, ChIP assays demonstrated that ZHX2 could pull down DNA fragments in the S100A14 promoter region. Whether ZHX2 directly or indirectly interacts with these DNA fragments still needs to be elucidated. In addition, the NF- $\mathrm{kB}$ signalling pathway participates in PTC progression by regulating the EMT process [45]. It is possible that ZHX2 regulates thyroid cancer metastasis through multiple pathways. Nevertheless, our data demonstrated that ZHX2 inhibits thyroid cancer metastasis by repressing S100A14 expression at the transcriptional level.

In conclusion, we identified S100A14 as a new target of ZHX2 and revealed that ZHX2 bound to the S100A14 promoter to repress its transcription, which in turn 
inhibited thyroid cancer metastasis. Our findings expand the understanding of the molecular mechanism underlying thyroid cancer metastasis and provide a potentially effective target for thyroid cancer diagnosis and therapy.

\section{Abbreviations}

ZHX2: Zinc fingers homeoboxes; PTC: Papillary thyroid cancer; FTC: Follicular thyroid cancer; ATC: Anaplastic thyroid cancer; S100A14: S100 calcium binding protein A14; RT-qPCR: Quantitative real-time PCR; qPCR: Quantitative PCR; ChIP: Chromatin immunoprecipitation; IHC: Immunohistochemical; EMT: Epithelial mesenchymal transition.

\section{Supplementary Information}

The online version contains supplementary material available at https://doi. org/10.1186/s12935-022-02499-w.

Additional file 1: Table S1. The information of patients involved in this study.

Additional file 2: Table S2. Synthetic oligonucleotides.

Additional file 3: Table S3. The CBioPortal analysis negative correlation of ZHX2 expression with $\mathrm{S} 100$ family in thyroid cancer patients [Thyroid Carcinoma (TCGA, PanCancer Atlas)] listed in the table.

\section{Acknowledgements}

We thank Translational Medicine Core Facility of Shandong University for consultation and instrument availability that supported this work.

\section{Authors' contributions}

Conceptualization, $C M, X Y$, and $Y Z$; methodology, $Y Z, X Y$, and $M S$; formal analysis, $X Y$, and $Y Z$; investigation, $Y Z$; resources, $Y Z$, and $J$; writing-original draft preparation, $C M, X Y$, and $Y Z$; writing-review and editing, all authors; funding acquisition, $C M, X Y$, and JL. All authors read and approved the final manuscript.

\section{Funding}

This work was supported by grants from the National Natural Science Foundation of China (Key project 81830017, 81972819, 81702647), Taishan Scholar Foundation of Shandong Province (No. tspd20181201), Collaborative Innovation Center of Technology and Equipment for Biological Diagnosis and Therapy in Universities of Shandong, Natural Science Foundation of Shandong Province (No. ZR2020YQ57).

\section{Availability of data and materials}

The data that supports the findings of our study are available from the corresponding author upon reasonable request.

\section{Declarations}

\section{Ethics approval and consent to participate}

The study was conducted according to the guidelines of the Declaration of Helsinki, and approved by the local ethics committee (the Ethics Committee of School of Basic Medical Sciences, Shandong University).

\section{Consent for publication}

All authors agree with the content for publication.

\section{Competing interests}

The authors declare no competing interests.

\section{Author details}

${ }^{1}$ Key Laboratory for Experimental Teratology of Ministry of Education, Key Laboratory of Infection and Immunity of Shandong Province and Department of Immunology, School of Basic Medical Sciences, Cheeloo Medical College of Medicine, Shandong University, Jinan 250012, China. ${ }^{2}$ Department of Hernia and Abdominal Wall Surgery, General Surgery, Qilu Hospital, Cheeloo College of Medicine, Shandong University, Jinan 250012, China. ${ }^{3}$ Department of Cell Biology, School of Basic Medical Science, Cheeloo College of Medicine, Shandong University, Jinan 250012, China.

Received: 5 October 2021 Accepted: 30 January 2022

Published online: 12 February 2022

\section{References}

1. Rajoria S, Suriano R, Shanmugam A, Wilson YL, Schantz SP, Geliebter J, Tiwari RK. Metastatic phenotype is regulated by estrogen in thyroid cells. Thyroid. 2010;20(1):33-41.

2. Hu J, Yuan IJ, Mirshahidi S, Simental A, Lee SC, Yuan X. Thyroid carcinoma: phenotypic features, underlying biology and potential relevance for targeting therapy. Int J Mol Sci. 2021. https://doi.org/10.3390/ijms220419 50.

3. Cooper DS, Doherty GM, Haugen BR, Kloos RT, Lee SL, Mandel SJ, Mazzaferri EL, Mclver B, Pacini F, Schlumberger M, et al. Revised american thyroid association management guidelines for patients with thyroid nodules and differentiated thyroid cancer. Thyroid. 2009;19(11):1167-214.

4. Sherman SI, Brierley JD, Sperling M, Ain KB, Bigos ST, Cooper DS, Haugen BR, Ho M, Klein I, Ladenson PW, et al. Prospective multicenter study of thyroiscarcinoma treatment: initial analysis of staging and outcome. National Thyroid Cancer Treatment Cooperative Study Registry Group. Cancer. 1998;83(5):1012-21.

5. Kondo T, Ezzat S, Asa SL. Pathogenetic mechanisms in thyroid follicularcell neoplasia. Nat Rev Cancer. 2006;6(4):292-306.

6. Olsson M, Lindahl G, Ruoslahti E. Genetic control of alpha-fetoprotein synthesis in the mouse. J Exp Med. 1977;145(4):819-27.

7. Perincheri S, Dingle RW, Peterson ML, Spear BT. Hereditary persistence of alpha-fetoprotein and $\mathrm{H} 19$ expression in liver of BALB/CJ mice is due to a retrovirus insertion in the Zhx2 gene. Proc Natl Acad Sci USA. 2005:102(2):396-401.

8. Luan F, Liu P, Ma H, Yue X, Liu J, Gao L, Liang X, Ma C. Reduced nucleic ZHX2 involves in oncogenic activation of glypican 3 in human hepatocellular carcinoma. Int J Biochem Cell Biol. 2014;55:129-35.

9. Shen H, Luan F, Liu H, Gao L, Liang X, Zhang L, Sun W, Ma C. ZHX2 is a repressor of alpha-fetoprotein expression in human hepatoma cell lines. J Cell Mol Med. 2008;12(6b):2772-80.

10. Yue X, Zhang Z, Liang X, Gao L, Zhang X, Zhao D, Liu X, Ma H, Guo M, Spear BT, et al. Zinc fingers and homeoboxes 2 inhibits hepatocellular carcinoma cell proliferation and represses expression of Cyclins A and $\mathrm{E}$. Gastroenterology. 2012. https://doi.org/10.1053/j.gastro.2012.02.049.

11. Tian X, Wang Y, Li S, Yue W, Tian H. ZHX2 inhibits proliferation and promotes apoptosis of human lung cancer cells through targeting p38MAPK pathway. Cancer Biomarkers. 2020;27(1):75-84.

12. Cheng A, Guo X, Dai X, Wang Z. Upregulation of ZHX2 predicts poor prognosis and is correlated with immune infiltration in gastric cancer. FEBS Open Bio. 2021;11(6):1785-98.

13. Zhang J, Wu T, Simon J, Takada M, Saito R, Fan C, Liu XD, Jonasch E, Xie L, Chen X, et al. VHL substrate transcription factor ZHX2 as an oncogenic driver in clear cell renal cell carcinoma. Science. 2018;361(6399):290-5.

14. Schulten HJ, Hussein D, Al-Adwani F, Karim S, Al-Maghrabi J, Al-Sharif M, Jamal A, Bakhashab S, Weaver J, Al-Ghamdi F, et al. Microarray expression profiling identifies genes, including cytokines, and biofunctions, as diapedesis, associated with a brain metastasis from a papillary thyroid carcinoma. Am J Cancer Res. 2016;6(10):2140-61.

15. Chen QK, Lee K, Radisky DC, Nelson CM. Extracellular matrix proteins regulate epithelial-mesenchymal transition in mammary epithelial cells. Differentiation. 2013;86(3):126-32.

16. Ferrari SM, Fallahi P, Elia G, Ragusa F, Ruffilli I, Paparo SR, Antonelli A. Thyroid autoimmune disorders and cancer. Semin Cancer Biol. 2020;64:135-46.

17. Dongre A, Weinberg RA. New insights into the mechanisms of epithelialmesenchymal transition and implications for cancer. Nat Rev Mol Cell Biol. 2019;20(2):69-84.

18. Mack GS, Marshall A. Lost in migration. Nat Biotechnol. 2010;28(3):214-29.

19. Bresnick AR, Weber DJ, Zimmer DB. S100 proteins in cancer. Nat Rev Cancer. 2015;15(2):96-109. 
20. Wang X, Yang J, Qian J, Liu Z, Chen H, Cui Z. S100A14, a mediator of epithelial-mesenchymal transition, regulates proliferation, migration and invasion of human cervical cancer cells. Am J Cancer Res. 2015;5(4):1484-95.

21. Jiang S, Zhu Y, Chen Z, Huang Z, Liu B, Xu Y, Li Z, Lin Z, Li M. S100A14 inhibits cell growth and epithelial-mesenchymal transition (EMT) in prostate cancer through FAT1-mediated Hippo signaling pathway. Hum Cell. 2021;34(4):1215-26.

22. Li X, Wang M, Gong T, Lei X, Hu T, Tian M, Ding F, Ma F, Chen H, Liu Z. A S100A14-CCL2/CXCL5 signaling axis drives breast cancer metastasis. Theranostics. 2020;10(13):5687-703.

23. Zhang C, Lv B, Yi C, Cui X, Sui S, Li X, Qi M, Hao C, Han B, Liu Z. Genistein inhibits human papillary thyroid cancer cell detachment, invasion and metastasis. J Cancer. 2019;10(3):737-48.

24. Wu Z, Ma H, Wang L, Song X, Zhang J, Liu W, Ge Y, Sun Y, Yu X, Wang Z, et al. Tumor suppressor ZHX2 inhibits NAFLD-HCC progression via blocking LPL-mediated lipid uptake. Cell Death Differ. 2019. https://doi.org/10. 1038/s41418-019-0453-z

25. Takenoue T, Kitayama J, Takei Y, Umetani N, Matsuda K, Nita ME, Hatano K, Tsuruo T, Nagawa H. Characterization of dihydropyrimidine dehydrogenase on immunohistochemistry in colon carcinoma, and correlation between immunohistochemical score and protein level or messenger RNA expression. Ann Oncol. 2000;11(3):273-9.

26. Kang Y, Massagué J. Epithelial-mesenchymal transitions: twist in development and metastasis. Cell. 2004;1 18(3):277-9.

27. Saleem M, Kweon M-H, Johnson JJ, Adhami VM, Elcheva I, Khan N, Bin Hafeez B, Bhat KMR, Sarfaraz S, Reagan-Shaw S, et al. S100A4 accelerates tumorigenesis and invasion of human prostate cancer through the transcriptional regulation of matrix metalloproteinase 9. Proc Natl Acad Sci USA. 2006;103(40):14825-30.

28. Ji Y-F, Huang H, Jiang F, Ni R-Z, Xiao M-B. S100 family signaling network and related proteins in pancreatic cancer (Review). Int J Mol Med. 2014;33(4):769-76.

29. Goyette J, Yan WX, Yamen E, Chung YM, Lim SY, Hsu K, Rahimi F, Di Girolamo N, Song C, Jessup W, et al. Pleiotropic roles of S100A12 in coronary atherosclerotic plaque formation and rupture. I Immunol. 2009;183(1):593-603.

30. Dreos R, Ambrosini G, Groux R, Cavin Périer R, Bucher P. The eukaryotic promoter database in its 30th year: focus on non-vertebrate organisms. Nucleic Acids Res. 2017;45(D1):D51-5.

31. Dreos R, Ambrosini G, Périer RC, Bucher P. The eukaryotic promoter database: expansion of EPDnew and new promoter analysis tools. Nucleic Acids Res. 2015;43:D92-6.

32. Siegel RL, Miller KD, Jemal A. Cancer statistics, 2018. CA Cancer J Clin. 2018. https://doi.org/10.3322/caac.21442.

33. Nagaiah G, Hossain A, Mooney CJ, Parmentier J, Remick SC. Anaplastic thyroid cancer: a review of epidemiology, pathogenesis, and treatment. J Oncol. 2011;2011:542358.

34. Ma H, Yue X, Gao L, Liang X, Yan W, Zhang Z, Shan H, Zhang H, Spear BT, $\mathrm{Ma}$ C. ZHX2 enhances the cytotoxicity of chemotherapeutic drugs in liver tumor cells by repressing MDR1 via interfering with NF-YA. Oncotarget. 2015;6(2):1049-63.

35. Zhu L, Ding R, Yan H, Zhang J, Lin Z. ZHX2 drives cell growth and migration via activating MEK/ERK signal and induces Sunitinib resistance by regulating the autophagy in clear cell renal cell carcinoma. Cell Death Dis. 2020;11(5):337.

36. Liu Y, Ma D, Ji C. Zinc fingers and homeoboxes family in human diseases. Cancer Gene Ther. 2015;22(5):223-6.

37. Pietas A, Schlüns K, Marenholz I, Schäfer BW, Heizmann CW, Petersen I. Molecular cloning and characterization of the human S100A14 gene encoding a novel member of the $\mathrm{S} 100$ family. Genomics. 2002:79(4):513-22.

38. Basnet S, Sharma S, Costea DE, Sapkota D. Expression profile and functional role of S100A14 in human cancer. Oncotarget. 2019;10(31):2996-3012.

39. Al-Ismaeel Q, Neal CP, Al-Mahmoodi H, Almutairi Z, Al-Shamarti I, Straatman K, Jaunbocus N, Irvine A, Issa E, Moreman C, et al. ZEB1 and IL6/11-STAT3 signalling cooperate to define invasive potential of pancreatic cancer cells via differential regulation of the expression of $\mathrm{S} 100$ proteins. Br J Cancer. 2019;121(1):65-75.
40. Zhu M, Wang H, Cui J, Li W, An G, Pan Y, Zhang Q, Xing R, Lu Y. Calciumbinding protein $\mathrm{S} 100 \mathrm{~A} 14$ induces differentiation and suppresses metastasis in gastric cancer. Cell Death Dis. 2017;8(7):e2938.

41. Tripathi D, Kulkarni S. Butein exhibits anti-tumor effect through intrinsic pathway of apoptosis, vimentin proteolysis, and inhibition of cancer stem cell population in the human papillary thyroid cancer cell line. Toxicol In Vitro. 2021. https://doi.org/10.1016/j.tiv.2021.105244.

42. Shakib H, Rajabi S, Dehghan MH, Mashayekhi FJ, Safari-Alighiarloo N, Hedayati M. Epithelial-to-mesenchymal transition in thyroid cancer: a comprehensive review. Endocrine. 2019;66(3):435-55.

43. Jiang S, Zhu Y, Chen Z, Huang Z, Liu B, Xu Y, Li Z, Lin Z, Li M. S100A14 inhibits cell growth and epithelial-mesenchymal transition (EMT) in prostate cancer through FAT1-mediated Hippo signaling pathway. Hum Cell. 2021;34(4):1215-26.

44. Kawata H, Yamada K, Shou Z, Mizutani T, Yazawa T, Yoshino M, Sekiguchi T, Kajitani T, Miyamoto K. Zinc-fingers and homeoboxes (ZHX) 2, a novel member of the ZHX family, functions as a transcriptional repressor. Biochem J. 2003;373(Pt 3):747-57.

45. Lv N, Liu F, Cheng L, Liu F, Kuang J. The expression of transcription factors is different in papillary thyroid cancer cells during TNF—a induced EMT. J Cancer. 2021;12(9):2777-86.

\section{Publisher's Note}

Springer Nature remains neutral with regard to jurisdictional claims in published maps and institutional affiliations.

Ready to submit your research? Choose BMC and benefit from

- fast, convenient online submission

- thorough peer review by experienced researchers in your field

- rapid publication on acceptance

- support for research data, including large and complex data types

- gold Open Access which fosters wider collaboration and increased citations

- maximum visibility for your research: over 100M website views per year

At BMC, research is always in progress.

Learn more biomedcentral.com/submissions 